\title{
Olive Oil-Supplemented Lipid Emulsion Induces CELF1 Expression and Promotes Apoptosis in Caco-2 Cells
}

\author{
Jun-Kai Yan ${ }^{a, b}$ Jie Zhu ${ }^{a, b}$ Zi-Zhen Gong ${ }^{a, b}$ Jie Wen ${ }^{a, b} \quad$ Yong-Tao Xiao a,b \\ Tian Zhang ${ }^{a, b}$ Wei Caia,
}

${ }^{a}$ Xin Hua Hospital affiliated to Shanghai Jiao Tong University School of Medicine, bShanghai Key Laboratory of Pediatric Gastroenterology and Nutrition, Shanghai Institute for Pediatric Research, Shanghai, China

\section{Key Words}

Parenteral nutrition • Olive oil-supplemented lipid emulsion • CELF1 • Apoptosis • Caco-2

\begin{abstract}
Background and Aims: Parenterally-administered lipid emulsion (LE) is a key cause of enterocyte apoptosis under total parenteral nutrition, yet the pathogenesis has not been fully understood. CUGBP, Elav-like family member 1 (CELF1) has been recently identified as a crucial modulator of apoptosis, and thus this study sought to investigate its role in the LE-induced apoptosis in vitro. Methods: Caco-2 cells were used as an in vitro model. The cells were treated with varying LEs derived from soybean oil, olive oil or fish oil, and changes in the apoptosis and CELF1 expression were assessed. Rescue study was performed using transient knockdown of CELF1 with specific siRNA prior to LE treatment. Regulation of CELF1 by LE treatment was studied using quantitative real-time PCR and Western blotting. Results: All the LEs upregulated CELF1expression and induced apoptosis, but only olive oil-supplemented lipid emulsion (OOLE)-induced apoptosis was attenuated by depletion of CELF1. Up-regulation of apoptosis-inducing factor (AIF) was involved in OOLE-induced CELF1 dependent apoptosis. The protein expression of CELF1 was up-regulated by OOLE in a dose- and time-dependent manner, but the mRNA expression of CELF1 was unchanged. Analysis by polysomal profiling and nascent protein synthesis revealed that the regulation of CELF1 by OOLE treatment was mediated by directly accelerating its protein translation. Conclusion: OOLE-induces apoptosis in Caco-2 cells partially through up-regulation of CELF1.
\end{abstract}

\section{Introduction}

Total parenteral nutrition (TPN) provides all nutrition intravenously for those patients who are unable to tolerate enteral nutrition (EN). Despite its advantages, there is a substantial body of evidence that TPN leads to intestinal atrophy partially due to increased 
enterocyte apoptosis [1-3]. Though the pathogenesis has not been fully understood, by using a mouse model of TPN, some possible mechanisms have been reported, including impaired $\beta$-catenin signaling pathway [4], decline in phosphatidylinositol 3-kinase/Akt activity [5] and responses to pro-inflammatory cytokines $[6,7]$. However, whether these changes are attributed to deprivation of enteral nutrition or administration of parenteral nutrition (PN) remains elusive. Recently, an in vivo study has demonstrated that enterocyte apoptosis in a mouse model of TPN was dependent on the type of lipid emulsion (LE), indicating that LE per se might be one of the key causes of enterocyte apoptosis under TPN support [8]. Currently, commercially available LEs with various composition of fatty acids include: fish oil-derived lipid emulsion (FOLE), soybean oil-derived lipid emulsion (SOLE) and 80\% olive oil-supplemented lipid emulsion (OOLE), which predominantly contains n-3, n-6 and n-9 unsaturated fatty acids, respectively. Although the impacts of varying LEs on cell apoptosis have been studied previously [9-11], the underlying mechanisms have not been fully understood. Most importantly, key proteins that trigger, mediate or modulate the apoptotic process in response to LE treatment are rarely characterized, leading to limited approaches for preventing TPN-induced intestinal atrophy in clinical practice.

CUGBP, Elav-like family member 1 (CELF1) is one of the RNA binding proteins that function as potent posttranslational regulators of gene expression [12]. It was originally found to interact with RNA CUG repeats expanded in patients with myotonic dystrophy type 1 , and has been recently well characterized for its contribution to the control of proliferation and apoptosis in a variety of cell types. Interestingly, this function of CELF1 appears not monotonous, but flexible, depending on the cell types. For example, by using rat intestinederived IEC- 6 cells and human Caco- 2 cells, it has been revealed that CELF1 may act as a potent anti-proliferation regulator in enterocytes $[13,14]$. Conversely, it functions as a proproliferation regulator in a variety of cancer cells, including oral cancer cells and laryngeal cancer cells $[15,16]$. These findings aroused our great interest in the role of CELF1 in LEinduced apoptosis in enterocytes.

In this study, we used human gut-derived Caco- 2 cells as a model, which has been approved suitable for studying intestinal homeostasis and studying the ingredients of parenteral nutrition in vitro [17]. We investigated the expression of CELF1 in the cells exposed to varying LEs, and explored the possible mechanisms linking CELF1 and LE-induced cell death. These results may be helpful to better understand the pathogenesis of enterocyte apoptosis under TPN support.

\section{Material and Methods}

\section{Chemicals and reagents}

Cell culture reagents were purchased from Life Technologies (Shanghai, China). Rabbit anti-GAPDH and mouse anti-CELF1 were purchased from Santa Cruz Biotechnology (Shanghai, China). Mouse anti-Ecadherin was purchased from BD (Shanghai, China). Rabbit anti-caspase-3, 7, and cleaved caspase-3, 7 were purchased from CST (Shanghai, China). Rabbit anti-AIF was purchased from Proteintech (Shanghai, China). Horseradish peroxidase conjugated anti-mouse and anti-rabbit IgG were purchased from CST (Shanghai, China). All other chemicals were purchased from Sigma (Shanghai, China).

\section{Cell culture}

Caco- 2 cells (American Type Culture Collection, USA) were cultured in Dulbecco's modified Eagle medium supplemented with $10 \%$ fetal bovine serum, at $37{ }^{\circ} \mathrm{C}$ in a humidified atmosphere containing $5 \%$ $\mathrm{CO}_{2}$. The medium was changed every two days.

Plasmid construction, RNA interference and transfection

CELF1 expression vector was purchased from Origene (Rockville, MD). The siRNAs targeting CELF1 were purchased from Santa Cruz Biotechnology (sc-38251). Transient transfections were performed using Lipofectamine Reagent (Life Technology) following the manufacturer's protocol.

\section{KARGER}




\section{Cellular Physiology Cell Physiol Biochem 2017;41:711-721 and Biochemistry Published \begin{tabular}{l|l} 
DOI: 10.1159/000458430 & $\begin{array}{l}\text { (c) } 2017 \text { The Author(s). Published by S. Karger AG, Basel } \\
\text { www.karger.com/cpb }\end{array}$
\end{tabular}}

Yan et al.: CELF1 and OOLE-Induced Apoptosis

\section{Cell treatment}

Experimental LEs were derived from commercial products as follows: SOLE (Lipofundin LCT/MCT, 20\%, Baxter Healthcare, China), OOLE (Clinoleic, 20\%, Baxter Healthcare, China), FOLE (Omegaven, 10\%, Fresenius Kabi, China). Caco-2 cells were exposed to 1\% OOLE, 1\% SOLE or 2\% FOLE for 48 hours to test the effect of varying LEs on the expression of CELF1 and cell apoptosis. The concentration of FOLE was set at $2 \%$ because it was derived from Omegaven which contains half the amount of fatty acids in Lipofundin and Clinoleic. Cells were exposed to various concentrations of OOLE $(0.2 \%, 0.5 \%$, and $1 \% \mathrm{vol} / \mathrm{vol}$ dissolved in culture medium) for 1-48 hours to test the effect of OOLE dose and OOLE incubation time on CELF1 expression. In some experiments, treatment with cycloheximide (CHX, at $25 \mu \mathrm{g} / \mathrm{ml}$ ) and tumor necrosis factor $\alpha(\mathrm{TNF} \alpha$, at $20 \mathrm{ng} / \mathrm{ml}$ ) for 6 hours was set as a positive control for caspase-dependent apoptosis.

\section{Apoptosis assessment}

Apoptosis was quantified by flow cytometry using the FITC-Annexin V apoptosis detection kit according to the manufacturer's instructions. Briefly, cells were collected, washed with PBS, and resuspended in cold binding buffer. After incubation for $15 \mathrm{~min}$ in the dark at $4{ }^{\circ} \mathrm{C}$, cells were stained with FITC-Annexin V (final concentration, $1 \mu \mathrm{g} / \mathrm{ml}$ ) and PI (final concentration, $5 \mu \mathrm{g} / \mathrm{ml}$ ). Stained cells were analyzed with a FACSCalibur flow cytometer (BD Biosciences, USA). A total 1 $10^{4}$ cells were analyzed per sample.

\section{Quantitative real-time PCR}

Total RNA was extracted from Caco-2 cells using Trizol isolation method (life technology, USA). The sequences of primers used in this study were as follows: human CELF1: 5'-TCCTGCCGTTTGTTCATCGTT-3' (forward) and 5'- TTTCCCCTTCAGCAGTCGTTC-3' (reverse); human GAPDH: 5'- TATTGTTGCCATCAATGACCC -3 ' (forward) and 5'-ACTCCACGACGTACTCAGC-3' (reverse). Quantitative real-time PCR and data analysis were performed using PIK096 (Thermo, Germany).

\section{Western blot analysis}

Cells were washed twice with PBS and lysed on ice for 30 minutes in $100 \mu \mathrm{l}$ of RIPA buffer. Protein concentrations were determined using a BCA protein assay kit (Thermo, USA). Aliquots of the lysates (30 $\mu \mathrm{g}$ of protein) were loaded and immunodetection was performed with enhanced chemiluminescence detection system (GE Healthcare, USA).

\section{Immunofluorescence staining}

Cells were fixed in $4 \%$ formaldehyde at room temperature for $15 \mathrm{~min}$, and permeabilized with $0.2 \%$ Triton-X100 in PBS for 15 minutes. After blocking, cells were incubated with antibodies against CELF1 and E-cadherin at $4{ }^{\circ} \mathrm{C}$ overnight, followed by incubation with Alexa Fluor 488 or Alexa Fluor 594 conjugated secondary antibodies for $1 \mathrm{~h}$ at room temperature. Immunostaining were observed on a Leica DMI6000B fluorescence microscopy with LAS AF LITE image processing software (Leica, Germany).

\section{Protein stability assay}

Cells were treated with CHX $(0.1 \mathrm{mg} / \mathrm{ml})$ for $0,2,4$ and 8 hours. The protein abundance of CELF1 at $0 \mathrm{~h}$ time point was set as $100 \%$ and thereafter, the percentage of normalized CELF1 protein levels versus time was plotted.

\section{Analysis of newly synthesized protein}

Nascent CELF1 protein was detected by Click-iT protein analysis detection kit (Invitrogen) according to the manufacturer's protocol. Briefly, cells were incubated in methionine-free medium and then exposed to L-azidohomoalanine. After mixing of cell lysates with the reaction buffer containing biotin/alkyne reagent and $\mathrm{CuSO} 4$ for $20 \mathrm{~min}$, the biotinalkyne/azide-modified protein complex was pulled down using paramagnetic streptavidin-conjugated Dynabeads. The pull-down material was resolved by $10 \%$ SDS-PAGE and analyzed by Western blotting analysis using the antibody against CELF1 or GAPDH.

Polysomal profiling analysis

Polysomal profiling analysis was carried as described previously [18]. Briefly, cells were incubated for $15 \mathrm{~min}$ in CHX at $0.1 \mathrm{mg} / \mathrm{ml}$, and then collected and lysed on ice for $10 \mathrm{~min}$. Nuclei were pelleted $(10,000$ 
$\times \mathrm{g}, 10 \mathrm{~min}$ ), and the resulting supernatant was fractionated through a $10-50 \%$ linear sucrose gradient to fractionate cytoplasmic components. The eluted fractions were prepared with a fraction collector (Brandel, Gaithersburg, MD). The levels of individual mRNA in each fraction were quantified by real-time PCR, and their abundance represented as a percentage of the total mRNA in the gradient.

\section{Statistical analysis}

Data in the figures and text are expressed as means \pm standard deviation (S.D.) of at least three experiments each performed in triplicates. Statistical analysis employed Student's $t$ test for comparison of two means, and a one-way ANOVA for comparison of multiple groups. A significant difference between means was considered to be present when $p<0.05$.

\section{Results}

Experimental LEs increases CELF1 expression and induces caspase-independent apoptosis in Caco-2 cells

Cells were treated with varying LEs for 48 hours. As shown in Fig. 1A-B, all LEs led to a significant increase in the expression of CELF1 in Caco-2 cells. Though no significant alterations in the expression of cleaved caspase-3/7 were observed, flow cytometry analysis revealed that apoptotic rate was significantly increased by treatment with varying LEs. Treatment with CHX/TNF $\alpha$, which elicited obvious increase in both expression of cleaved caspase-3/7 and apoptotic rate, was set as positive control of caspase-dependent apoptosis (Fig. 1C). Collectively, these results suggested that experimental LEs induced caspaseindependent apoptosis in Caco-2 cells, accompanied with increased expression of CELF1.

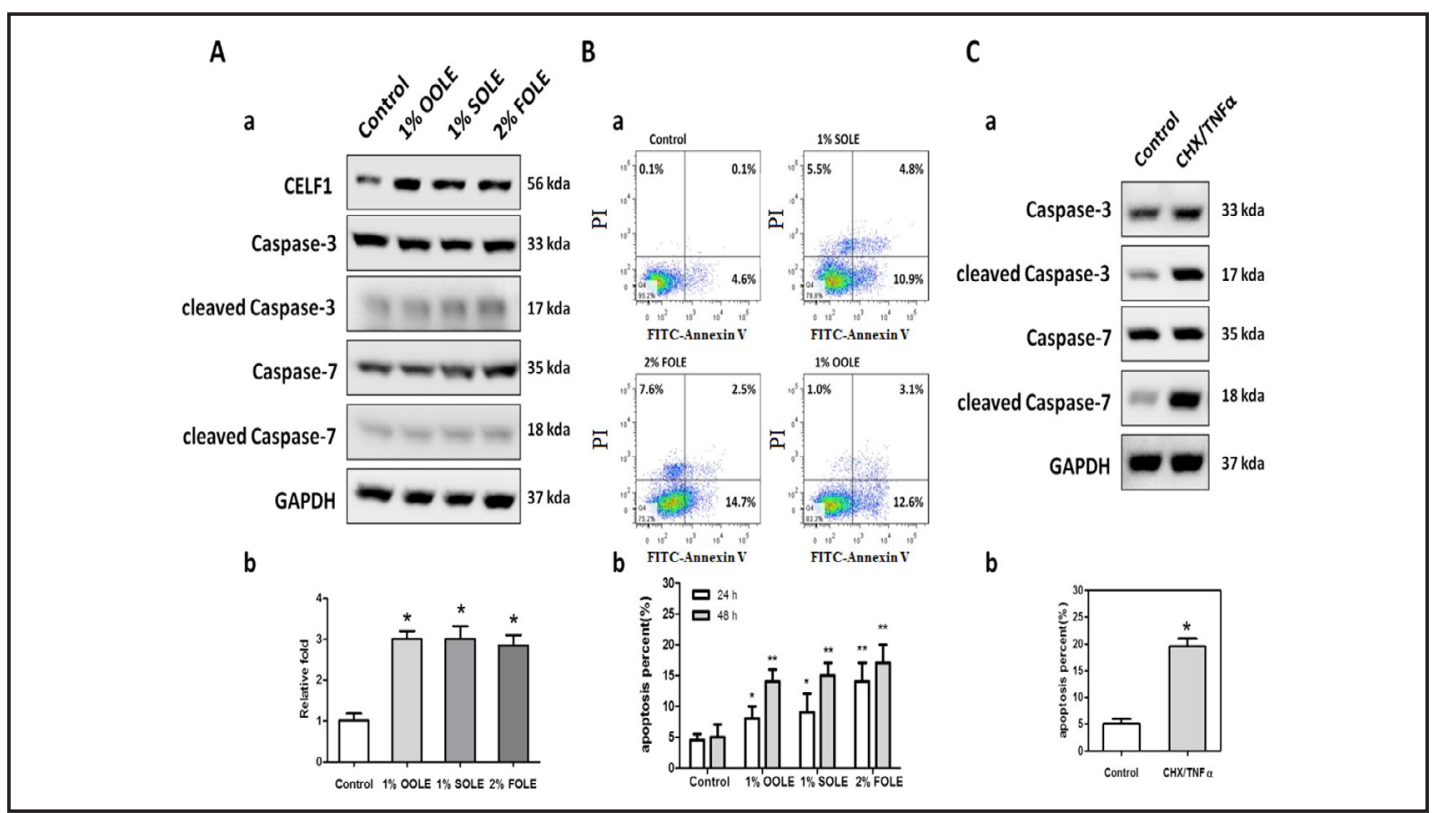

Fig. 1. Lipid emulsions (LEs) increased CELF1 expression and induced caspase-independent apoptosis in Caco-2 cells. (A) Representative blots of CELF1 levels, pro and cleaved form of caspase-3/7 in cells treated with varying LEs. Protein levels were measured at 48 hours post treatment, and relative fold of CELF1 was determined by analysis of band intensity. Values are mean \pm SD of data from three experiments. ${ }^{*}, p<0.05$. (B) Apoptosis in cells described in (A). Original dot plots were shown (a). The numbers shown in the lower or upper right indicate the percentage of early or late apoptotic cells, respectively. The graphs represent typical donors and are representative for three different experiments. Apoptotic percentages in cells treated with varying LEs for 24-48 hours were also presented within histograms (b). Values are mean \pm SD of data from three experiments. ${ }^{*}, \mathrm{p}<0.05$. **, $\mathrm{p}<0.01$ compared to control. (C) Representative blots of caspase-3/7 levels (a) and apoptotic rate (b) in cells treated with CHX $(25 \mu \mathrm{g} / \mathrm{ml}) / \mathrm{TNF} \alpha(20 \mathrm{ng} / \mathrm{ml})$ for 6 hours. Values are mean \pm SD of data from three experiments. ${ }^{*}, \mathrm{p}<0.05$. 
Fig. 2. Depletion of CELF1 partly attenuated OOLE-induced apoptosis in Caco-2 cells. (A) Representative blots of CELF1 levels in Caco-2 cells. Cells were transfected with CELF1 siRNA for 24 hours, followed by treatment with varying lipid emulsions for 48 hours. (B) The percentage of apoptosis in cells described in (A). Values are mean \pm SD of data from three experiments. ${ }^{*}, p<0.05$.

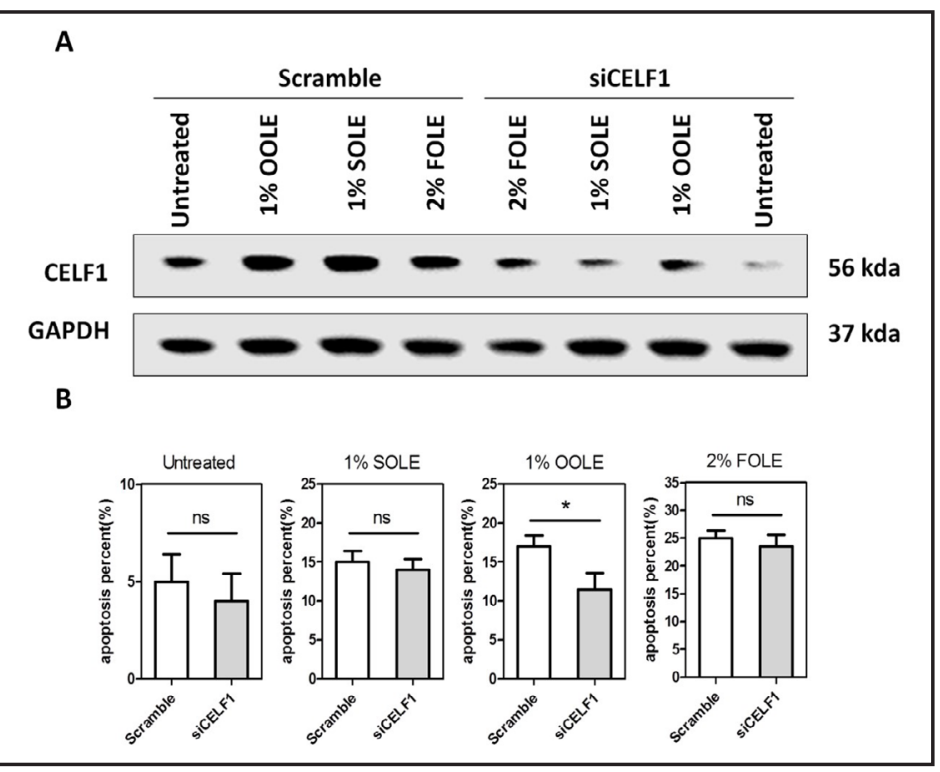

CELF1 is specially required for OOLE-induced apoptosis in Caco-2 cells

By depletion of CELF1 with specific siRNA, rescue studies were performed to further confirm the role of CELF1 in LE-induced apoptosis. As shown, up-regulation of CELF1 induced by varying LEs was markedly attenuated by depletion of CELF1 with specific siRNA (Fig. 2A). Depletion of CELF1 per se elicited no significant impact on apoptosis under untreated condition. However, OOLE-induced apoptosis was attenuated by CELF1 silencing, with no significant influence observed on the apoptosis induced by SOLE or FOLE (Fig. 2B). Taken together, these results suggested that CELF1 was specifically required for OOLE-induced apoptosis in Caco-2 cells.

AIF is involved in OOLE-induced CELF1-dependent apoptosis in Caco-2 cells

Ectopic over-expression of CELF1 induces AIF-dependent apoptosis in Caco-2 cells. The pro-apoptotic role of CELF1 in Caco- 2 cells was confirmed by transfection with CELF1 over-expression plasmids. As shown in Fig. 3A, 48 hours after transfection, the expression of CELF1 was obviously increased in Caco-2 cells. Notably, AIF was also obviously increased, while no significant changes in the expression of cleaved caspase-3/7 were observed. Taken together with the flow cytometry analysis which revealed a significant increase in the apoptotic rate of the cells over-expressing CELF1 (Fig. 3B), these results suggested that AIF may be involved in the apoptosis induced by ectopic over-expression of CELF1 in Caco2 cells. Furthermore, the expression of AIF was determined in the cells exposed to OOLE treatment and OOLE treatment in the absence of CELF1. As shown, depletion of CELF1 per se elicited no significant impact on AIF expression under untreated condition. However, it was significantly increased in the cells exposed to OOLE treatment, which was abolished by depletion of CELF1 (Fig. 3C). Collectively, these results indicated that AIF is involved in OOLE-induced CELF1-dependent apoptosis in Caco-2 cells, instead of caspases (Fig. 3D).

\section{Effect of OOLE treatment on the expression and subcellular localization of CELF1}

As shown, CELF1 protein levels were markedly increased on OOLE treatment in a dose-dependent manner (by approximately 1.1 fold at $0.2 \%, 1.6$ fold at $0.5 \%$ and 2.8 fold at $1 \%$ ). Since treatment with 1\% OOLE elicited the most significant impact on CELF1 expression, this concentration was further used in the time course study. As can be seen, OOLE treatment time-dependently increased OOLE protein levels. Notably, OOLE-induced up-regulation of CELF1 expression was already evident at 4 hours after treatment, which suggested a rapid mechanism for CELF1 regulation responding to OOLE may exist (Fig. 4A). Interestingly, inconsistent with the protein levels of CELF1, the mRNA levels of CELF1 were 


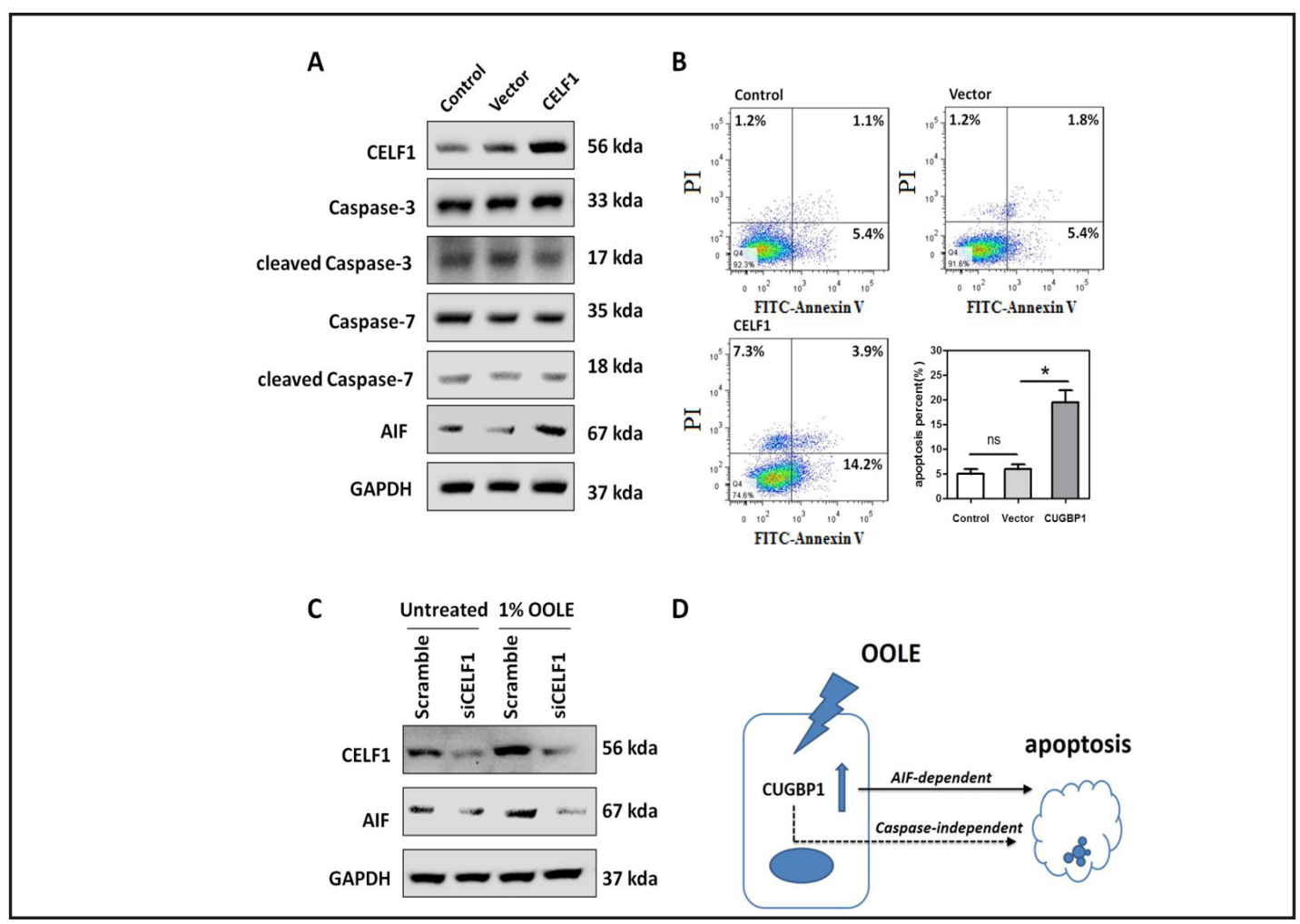

Fig. 3. AIF was involved in OOLE-induced CELF1-dependent apoptosis in Caco-2 cells. (A) Representative blots of AIF levels in cells transfected with plasmids over-expressing CELF1 for 48 hours. Note that a predominate increase in AIF expression was observed in the cells over-expressing CELF1, while cleaved products of caspase 3/7 were unchanged. (B) Original dot plots representing apoptosis in cells described in (A). The numbers shown in the lower or upper right indicate the percentage of early or late apoptotic cells, respectively. The graphs represent typical donors and are representative for three different experiments. Apoptotic percentages were also presented within histograms. (C) Representative blots of AIF levels in the cells exposed to OOLE treatment and OOLE treatment in the absence of CELF1. Note that AIF was significantly up-regulated after OOLE treatment, which was abolished by depletion of CELF1. (D) Schematic representation showing that OOLE induces CELF1 expression, leading to AIF-dependent apoptosis in Caco-2 cells.

not obviously affected by OOLE treatment (Fig. 4B). Moreover, immunofluorescence staining demonstrated that up-regulation of CELF1 expression was evident after OOLE treatment, but its predominant nuclear localization was not affected (Fig. 4C). These results suggested that CELF1 was up-regulated by OOLE treatment in a dose- and time-dependent manner, and this regulation may occur at the post-transcriptional level.

\section{Up-regulation of CELF1 does not involve protein degradation}

As indicated in Fig. 4, up-regulation of CELF1 by OOLE was evident only on protein levels. As elevated protein abundance could be resulted from either decrease in protein degradation or increase in protein translation, to test this, protein stability assay was performed. As shown in Fig. 5, no significant differences in protein degradation rates of CELF1 were observed between control cells and OOLE-treated cells, suggesting that protein degradation was not involved in the regulation of CELF1 responding to OOLE treatment.

OOLE-induced regulation of CELF1 is mediated by accelerating its protein translation

To examine whether regulation of CELF1 by OOLE is mediated by enhancing its translation, mRNA distribution in polysomes and nascent protein synthesis were analyzed. As shown, though OOLE treatment did not affect global polysomal profiles (Fig. 6B), relative 
Fig. 4. Effect of OOLE treatment on the expression and subcellular localization of CELF1. (A) Representative blots of CELF1 protein levels in Caco-2 cells treated with OOLE. Cells in (a) were treated with OOLE at $0.2 \%, 0.5 \%$ and $1 \%$ doses for 48 hours. Cells in (b) were treated with 1\% OOLE for 1, 2, 4, 24 and 48 hours. The protein expression of CELF1 was increased by 00LE treatment in a dose- and time-dependent manner. Relative fold was determined by analysis of band intensity. Values are mean \pm SD of data from three experiments.*, p<0.05. (B) CELF1 mRNA levels in Caco-2 cells treated with OOLE. Cells were treated as described in (A) and CELF1 mRNA levels were quantified by real-time PCR. Note that the mRNA expression of CELF1 was unchanged upon OOLE treatment. Values are mean \pm SD of data from three experiments. (C) Representative images of CELF1 staining in Caco-2 cells. Cells were treated with OOLE for 4 hours. Notably, a significant increase in CELF1 (green) expression was observed upon OOLE treatment while the nuc-

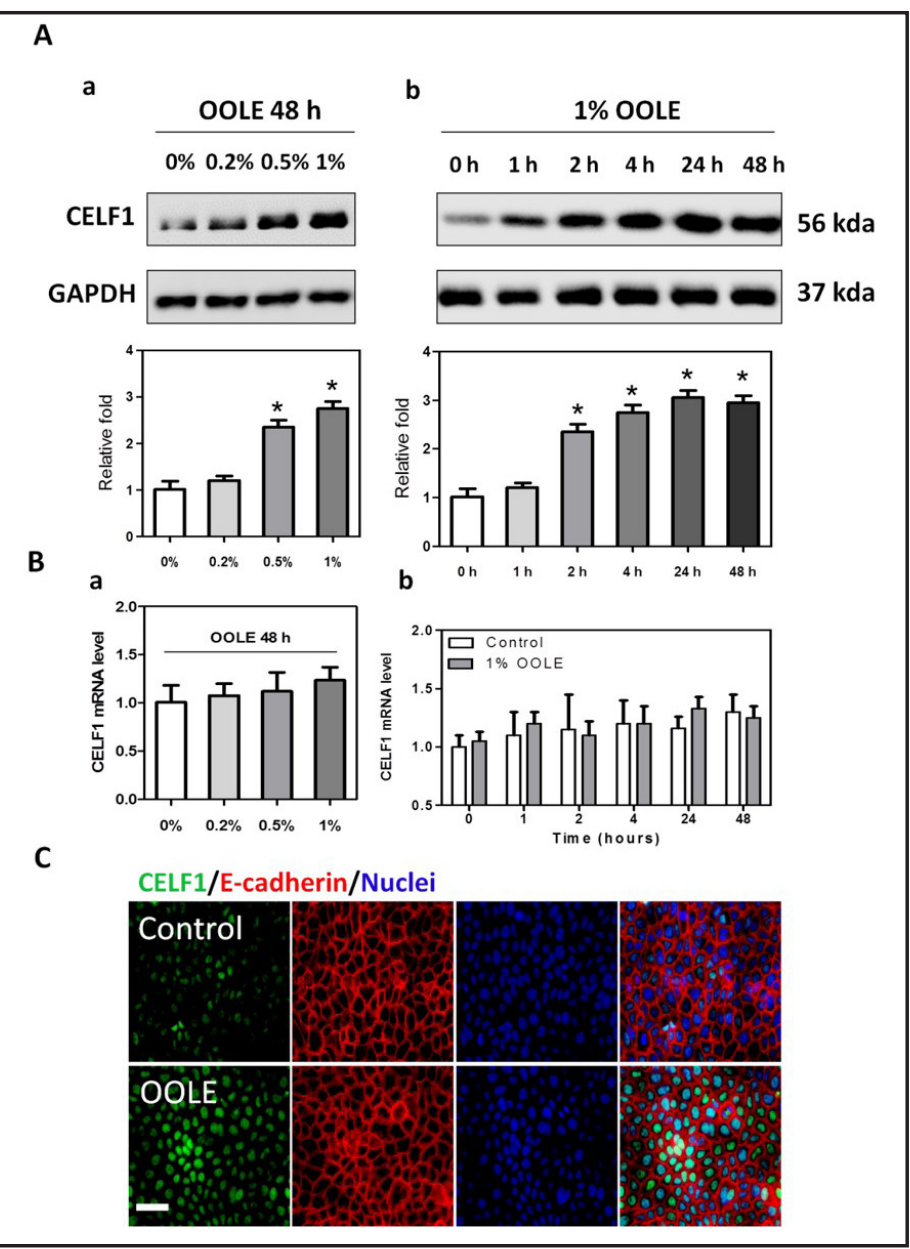
lear localization of CELF1 was unchanged. Cell membrane was labeled with E-cadherin (red). Scale bar=75 $\mu \mathrm{m}$. Three experiments were performed that showed similar results.

distribution of CELF1 mRNA showed that the abundance of CELF1 transcripts associated with actively low-translating fractions 1-5 decreased dramatically in OOLE-treated cells compared with control, with a significant shift of CELF1 transcripts to actively hightranslating fractions 6-10. In contrast, GAPDH mRNA distributed similarly in the two groups (Fig. 6C). Consistently, newly synthesized CELF1 protein increased significantly in OOLEtreated cells (by approximately 2.5 fold, $\mathrm{p}<0.05$ ), compared with control cells. No changes in nascent GAPDH synthesis were observed after OOLE treatment. Taken together, these results suggested that OOLE-induced regulation of CELF1 was mediated by directly accelerating its protein translation.

\section{Discussion}

A rodent study has recently demonstrated that TPN-associated intestinal atrophy is related to the type of LEs administrated intravenously [8]. Striking differences were noted in enterocyte apoptosis and proliferation when three commercial LEs were used, including SOLE, OOLE and SMOF (a combination of soybean oil, medium-chain triglycerides, olive oil and fish oil emulsion). Though differences in the pro-inflammatory cytokine state within the intestinal mucosa might be a reasonable explanation, the question is whether such possibility may exist that LEs directly act on enterocytes as exogenous stimuli and induce 


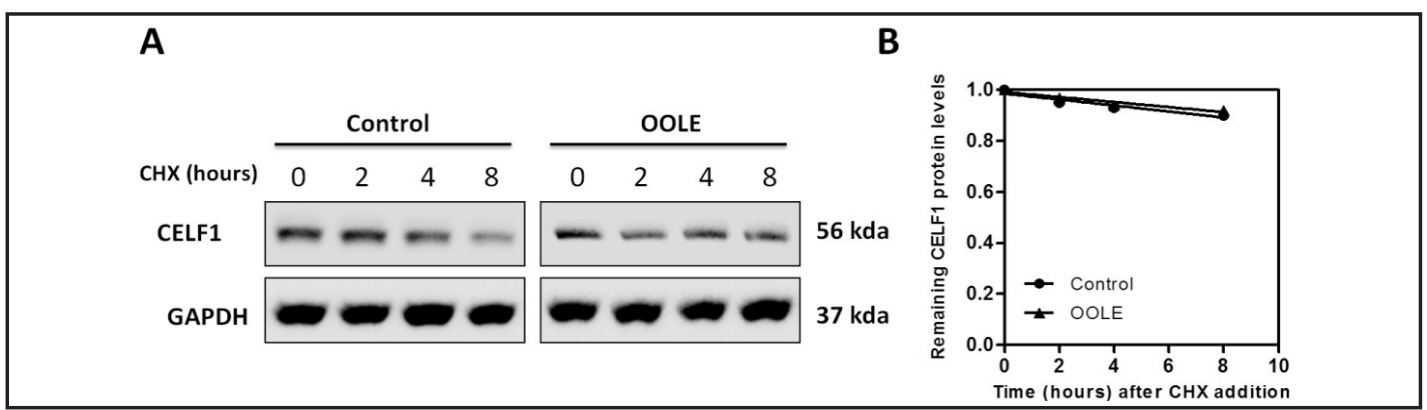

Fig. 5. CELF1 protein stability was unchanged by OOLE treatment. (A) Representative blots of CELF1 levels. Caco-2 cells were treated with CHX $(0.1 \mathrm{mg} / \mathrm{ml})$ for 30 minutes, and then further exposed to $1 \%$ OOLE (containing CHX) for the indicated periods. (B) Protein levels of CELF1 measured by optical density of immunoblotting signals and calculated as described in methods. Three experiments were performed that showed similar results.

Fig. 6. Regulation of CELF1 by OOLE was mediated by accelerating its protein translation in the ribosomes. (A) A schematic of ribosome isolation and polysomal analysis by sucrose gradient sedimentation. (B, C) Polysomal profiles in Caco-2 cells. Cells were treated with OOLE for 4 hours. Global distribution of $40 \mathrm{~S}$ subunit, $60 \mathrm{~S}$ subunit, $80 \mathrm{~S}$ subunit and polysomes was shown in B: control cells (left) and OOLE-treated cells (right). Relative distribution of either CELF1 mRNAs (top) and GAPDH mRNAs (bottom) in each gradient fraction was shown in C. (D) Nascent protein synthesis in Caco-2 cells described in B and C. Newly translated CELF1 protein was determined by using CLICKiT protein synthesis kit, and

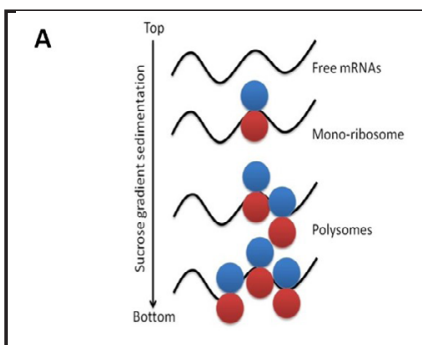

C
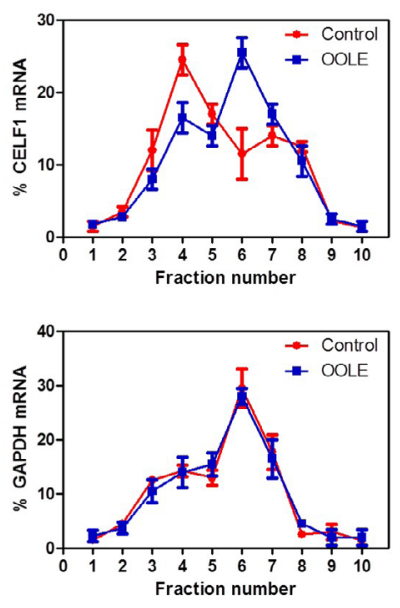

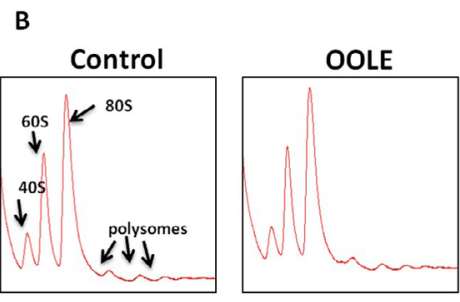

D

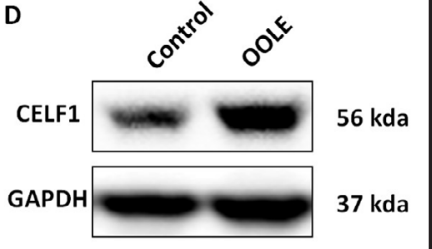
quantitative analysis of the immunoblotting signals was measured by densitometry. Values are mean \pm SD of data from three independent experiments. ${ }^{*} \mathrm{p}<0.05$, compared to control.

apoptosis, independent on those pro-inflammatory cytokines. Therefore, this study was designed to examine the direct impact of LEs on enterocytes in vitro, and to identify key proteins required for LE-induced apoptosis.

CELF1 is a multifunctional RNA-binding protein involved in the regulation of many posttranscriptional processes including alternative splicing, translation, de-adenylation, and mRNA degradation [19-22]. In this study, by using Caco-2 cells as an in vitro model, we obtained three major findings: 1) CELF1 is a pro-apoptotic and anti-proliferative regulator in Caco-2 cells; 2) AIF is a downstream effector of CELF1, contributing to the apoptotic process induced by OOLE; 3) Up-regulation of CELF1 by OOLE treatment is mediated by accelerating its translation. 
One of the intriguing findings in this study was the pro-apoptotic and anti-proliferative effect of CELF1 in Caco-2 cells (Fig. 3), though it appears to prevent apoptosis and stimulate proliferation in a variety of other cell types. Our results were consistent with those previously described by Hee Kyoung Chung et al, who revealed that ectopic over-expression of CELF1 inhibited cell proliferation while knockdown of CELF1 stimulated cell proliferation in IEC6 cells [13]. Given that both IEC- 6 and Caco-2 are intestinal epithelial cells, we suppose that the function of CELF1 in enterocytes may be distinct from that in other cell types. Moreover, our results revealed that CELF1-induced apoptosis seems to be mediated via caspase-independent but AIF-dependent pathway. A number of studies have demonstrated that changes in AIF may cause DNA damage and lead to caspase-independent apoptosis, contributing to the development of various diseases $[23,24]$. In this study, though it has been indicated that AIF is one of the downstream effectors of CELF1 mediating the apoptotic process, it is still not clear whether CELF1 can physically interact with AIF mRNA and posttranscriptionally regulate its expression or not. Therefore, it will be of great importance to be elucidated in future investigations.

Given that CELF1 has been confirmed as a pro-apoptotic regulator, we investigated whether CELF1 participated in the LE-induced enterocyte apoptosis. One aspect requires comment. The initial concentration of LEs used in this study was referenced to the in vitro study conducted by C. Guzy group [17]. In that study, 5\% PN containing approximately $20 \%$ of lipid was used, suggesting that the final concentration of LE was approximately $1 \%$. Therefore, 1\% LE was initially used in this study. The results revealed that all of the three LEs increased CELF1 expression and induced cell apoptosis (Fig. 1). Although surprising, only apoptosis induced by OOLE was attenuated by depletion of CELF1, suggesting CELF1 might be specifically important for OOLE-induced apoptosis (Fig. 2). Why depletion of CELF1 was effective only in OOLE-induced apoptosis? One of the reasonable explanations is that the capacity of LEs to induce apoptosis is distinct: generally FOLE $>$ SOLE $\geq 0 O L E$, according to their direct impacts reported in previous studies $[9,10,25]$. Blocking of a CELF1-dependent pathway may be sufficient to attenuate "mild" apoptosis stimuli like OOLE, but not so that effective for "severe" apoptosis stimuli like FOLE or SOLE. Therefore, we characterized CELF1 as a potential therapeutic target against OOLE-induced apoptosis.

Furthermore, we studied the mechanism by which OOLE induced CELF1 expression. Our results indicated that a post-transcriptional mechanism may be involved in the regulation of CELF1 expression, for the protein levels of CELF1 has been already evident within four hours after OOLE treatment while its mRNA levels were unchanged (Fig. 4). By studying polysomal profile and nascent protein synthesis, we found a significant shift of CELF1 transcripts to high-translating polysomes after OOLE treatment and a markedly increase in newly synthesized CELF1 proteins (Fig. 6). This rapid regulation of CELF1 suggested its capacity in triggering the apoptosis progress in the initial event responding to OOLE treatment. However, one of the limitations in this study is that the precise mechanism whereby CELF1 mediated the enterocyte apoptosis has not been fully determined. Therefore, it would be of great significance to further explore the upstream signaling pathway of CELF1, as well as its downstream targets in future studies.

In conclusion, our results suggested that OOLE-induces apoptosis in Caco-2 cells partially through up-regulation of CELF1. We are currently undertaking in vivo studies to further confirm the role of CELF1 in OOLE-induced apoptosis in enterocytes, and its contribution to TPN-induced intestinal atrophy.

\section{Acknowledgements}

This work was funded by Shanghai Municipal Health and Family Planning Commission (20144Y0087) and Ph.D Programs Foundation of Shanghai Jiaotong University School of Medicine (BXJ201620). 


\section{Cellular Physiology Cell Physiol Biochem 2017;41:711-721

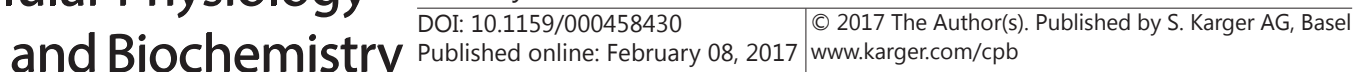 \\ Yan et al.: CELF1 and OOLE-Induced Apoptosis}

\section{Disclosure Statement}

The authors have declared no conflict of interest.

\section{References}

1 Van Gossum A, Cabre E, Hebuterne X, Jeppesen P, Krznaric Z, Messing B, Powell-Tuck J, Staun M, Nightingale J: ESPEN Guidelines on Parenteral Nutrition: Gastroenterology. Clin Nutr 2009;28:415-427.

- D'Antiga L, Goulet O: Intestinal failure in children: The European view. J Pediatr Gastroenterol Nutr 2013;56:118-126.

-3 Berger MM: The 2013 Arvid Wretlind lecture: Evolving concepts in parenteral nutrition. Clin Nutr 2014;33:563-570.

- Feng Y, Sun X, Yang H, Teitelbaum DH: Dissociation of E-cadherin and beta-catenin in a mouse model of total parenteral nutrition: A mechanism for the loss of epithelial cell proliferation and villus atrophy. J Physiol 2009;587:641-654.

-5 Feng Y, McDunn JE, Teitelbaum DH: Decreased phospho-Akt signaling in a mouse model of total parenteral nutrition: A potential mechanism for the development of intestinal mucosal atrophy. Am J Physiol Gastrointest Liver Physiol 2010;298:G833-G841.

6 Feng Y, Teitelbaum DH: Epidermal growth factor/TNF- $\alpha$ transactivation modulates epithelial cell proliferation and apoptosis in a mouse model of parenteral nutrition. Am J Physiol Gastrointest Liver Physiol 2012;302:G236-G249.

7 Freeman JJ, Feng Y, Demehri FR, Dempsey PJ, Teitelbaum DH: TPN-associated intestinal epithelial cell atrophy is modulated by TLR4/EGF signaling pathways. FASEB J 2015;29:2943-2958.

8 Feng Y, Browner P, Teitelbaum DH: Effects on varying intravenous lipid emulsions on the small bowel epithelium in a mouse model of parenteral nutrition. JPEN J Parenter Enteral Nutr 2013;37:775-786.

9 Jordan A, Stein J: Effect of an omega-3 fatty acid containing lipid emulsion alone and in combination with 5-fluorouracil (5-FU) on growth of the colon cancer cell line Caco-2. Eur J Nutr 2003;42:324-331.

10 Llor X, Pons E, Roca A, Alvarez M, Mane J, Fernandez-Banares F, Gassull MA: The effects of fish oil, olive oil, oleic acid and linoleic acid on colorectal neoplastic processes. Clin Nutr 2003;22:71-79.

11 Sala-Vila A, Folkes J, Calder PC: The effect of three lipid emulsions differing in fatty acid composition on growth, apoptosis and cell cycle arrest in the HT-29 colorectal cancer cell line. Clin Nutr 2010;29:519-524.

12 Wang ET, Ward AJ, Cherone JM, Giudice J, Wang TT, Treacy DJ, Lambert NJ, Freese P, Saxena T, Cooper TA, Burge CB: Antagonistic regulation of mRNA expression and splicing by CELF and MBNL proteins. Genome Res 2015;25:858-871.

13 Chung HK, Rao JN, Zou T, Liu L, Xiao L, Gu H, Turner DJ, Yang P, Wang JY: Jnk2 deletion disrupts intestinal mucosal homeostasis and maturation by differentially modulating RNA-binding proteins HuR and CUGBP1. Am J Physiol Cell Physiol 2014;306:C1167-C1175.

14 Liu L, Ouyang M, Rao JN, Zou T, Xiao L, Chung HK, Wu J, Donahue JM, Gorospe M, Wang JY: Competition between RNA-binding proteins CELF1 and HuR modulates MYC translation and intestinal epithelium renewal. Mol Biol Cell 2015;26:1797-1810.

-15 Talwar S, Balasubramanian S, Sundaramurthy S, House R, Wilusz CJ, Kuppuswamy D, D'Silva N, Gillespie MB, Hill EG, Palanisamy V: Overexpression of RNA-binding protein CELF1 prevents apoptosis and destabilizes pro-apoptotic mRNAs in oral cancer cells. RNA Biol 2013;10:277-286.

16 Zhou Y, Ma H, Fang J, Lian M, Feng L, Wang R: Knockdown of CUG-binding protein 1 induces apoptosis of human laryngeal cancer cells. Cell Biol Int 2014;38:1408-1414.

17 Guzy C, Schirbel A, Paclik D, Wiedenmann B, Dignass A, Sturm A: Enteral and parenteral nutrition distinctively modulate intestinal permeability and T cell function in vitro. Eur J Nutr 2009;48:12-21.

-18 Yu TX, Rao JN, Zou T, Liu L, Xiao L, Ouyang M, Cao S, Gorospe M, Wang JY: Competitive binding of CUGBP1 and HuR to occludin mRNA controls its translation and modulates epithelial barrier function. Mol Biol Cell 2013;24:85-99.

19 Berger DS, Ladd AN: Repression of nuclear CELF activity can rescue CELF-regulated alternative splicing defects in skeletal muscle models of myotonic dystrophy. PLoS Curr 2012;4:N1305. 


\section{Cellular Physiology Cell Physiol Biochem 2017;41:711-721 and Biochemistry DOI: 10.1159/000458430 2017 (0) 2017 The Author(s). Published by S. Karger AG, Basel

Yan et al.: CELF1 and OOLE-Induced Apoptosis

20 Xiao Q Ford AL, Xu J, Yan P, Lee KY, Gonzales E, West T, Holtzman DM, Lee JM: Bcl-x pre-mRNA splicing regulates brain injury after neonatal hypoxia-ischemia. J Neurosci 2012;32:13587-13596.

21 Giudice J, Xia Z, Wang ET, Scavuzzo MA, Ward AJ, Kalsotra A, Wang W, Wehrens XH, Burge CB, Li W, Cooper TA: Alternative splicing regulates vesicular trafficking genes in cardiomyocytes during postnatal heart development. Nat Commun 2014;5:3603.

22 Katoh T, Hojo H, Suzuki T: Destabilization of microRNAs in human cells by 3' deadenylation mediated by PARN and CUGBP1. Nucleic Acids Res 2015;43:7521-7534.

-23 Yu Q, Lu Z, Tao L, Yang L, Guo Y, Yang Y, Sun X, Ding Q: ROS-Dependent neuroprotective effects of NaHS in ischemia brain injury involves the PARP/AIF pathway. Cell Physiol Biochem 2015;36:1539-1551.

-24 Kanemura S, Tsuchiya A, Kanno T, Nakano T, Nishizaki T: Phosphatidylinositol induces CaspaseIndependent apoptosis of malignant pleural mesothelioma cells by accumulating AIF in the nucleus. Cell Physiol Biochem 2015;36:1037-1048.

25 Sala-Vila A, Folkes J, Calder PC: The effect of three lipid emulsions differing in fatty acid composition on growth, apoptosis and cell cycle arrest in the HT-29 colorectal cancer cell line. Clin Nutr 2010;29:519-524. 